\title{
Option pricing for stochastic volatility model with infinite activity Lévy jumps
}

\author{
Xiaoli Gong* Xintian Zhuang \\ School of Business Administration, Northeastern University, Shenyang, 110169, China
}

\begin{abstract}
The purpose of this paper is to apply the stochastic volatility model driven by infinite activity Lévy processes to option pricing which displays infinite activity jumps behaviors and time varying volatility that is consistent with the phenomenon observed in underlying asset dynamics. We specially pay attention to three typical Lévy processes that replace the compound Poisson jumps in Bates model, aiming to capture the leptokurtic feature in asset returns and volatility clustering effect in returns variance. By utilizing the analytical characteristic function and fast Fourier transform technique, the closed form formula of option pricing can be derived. The intelligent global optimization search algorithm called Differential Evolution is introduced into the above highly dimensional models for parameters calibration so as to improve the calibration quality of fitted option models. Finally, we perform empirical researches using both time series data and options data on financial markets to illustrate the effectiveness and superiority of the proposed method.
\end{abstract}

Keywords: stochastic volatility; infinite activity Lévy process; Differential Evolution algorithm; option pricing

\section{Introduction}

Recently, considerable researches have been conducted on option pricing in stochastic volatility models. The improvements of our presented model over the prevailing are the introduction of infinite jump behaviors for underlying asset returns which are modeled by infinite activity Lévy processes, where they can capture asymmetry, leptokurtosis and thicker tail properties in returns in addition to characterizing persistence effect and heteroskedasticity effect in volatility. The Lévy processes driven stochastic volatility models incorporates jumps and stochastic volatility simultaneously, which capture not only jumps but also stochastic volatility in stock price dynamics. The Lévy process can be an infinite activity process that generates an infinite number of jumps within any time interval, based on which the closed form option pricing formula can be derived due to the attainability of analytical characteristic functions, or it can be a finite activity process such as compound Poisson processes. In this work, we specially pay attention to the case where the Lévy jumps numbers are infinite to capture both large and small jumps in stock returns.

The traditional B-S model assumes the dynamics of underlying asset returns follow a geometric Brownian motion with constant drift and volatility parameters, which contradicts the phenomenon persistently observed in financial markets. Several stylized facts about asset returns distributions are widely accepted which include asymmetry, leptokurtosis and thicker tail nature than Gaussian distributions [1,2]. Moreover, there is abundant evidence that volatility exhibits clustering and heteroskedasticity effect, leading to stochastic jumps in stock prices. It has been demonstrated by Ait-Sahalia and Jacod [3], Klingler [4] that stochastic volatility and jumps are inherent components of the stock price dynamics that play important roles in the explanation of the implied volatility smile in options. Many alternative models are developed to reflect the intrinsic characteristics of asset returns and volatility smile effects of option prices, as shown by Kou [5], Mozumder [6], Shi [7], and Abdelrazeq [8]. Therefore, when constructing models to price options, it is necessary to incorporate both stochastic volatility and jumps. Just as what Carr and $\mathrm{Wu}$ [9] have addressed that infinite activity Lévy processes can capture both large jumps and small jumps in stock price dynamics that are consistently inspected in financial markets.

The alternative approach to solve the heteroskedasticity and volatility clustering effect for returns variance is by applying stochastic volatility models [10,11]. Even though plentiful work has been done on jump-diffusion stochastic volatility models see, for example, Todorov [12], Andreas et al. [13], Huang et al. [14], they feature a counter-factual assumption that jumps rarely occur. Bates [15] refined the Heston [16] model through adding a normally distributed compound Poisson jump for stock price dynamics, with the returns variance following the Cox-Ingersoll-Ross (CIR) mean reverting process employed in Schoutens [17]. Hence, it is reasonable to substitute the finite jump components in the Bates model into other arbitrary infinite Lévy jumps. We particularly focus on three infinite pure jumps Lévy processes that will be added to the stochastic volatility model, which are Variance Gamma processes (VG hereafter) proposed by Madan and Carr [18], Normal Inverse Gaussian processes (NIG hereafter) proposed by Barndorff-Nielsen [19], Classical Tempered Stable processes (CTS hereafter) presented by Rosinski [20]. Noticeably, the CGMY process proposed by Carr et al. [21] is also a special case of infinite activity tempered stable process which is extensively studied by Kim and Rachev [22], Kuchler [23] and Zaevski [24]. Tempered stable processes are the products of the Lévy measure of $\alpha$ stable process multiplying different tempered functions. Although $\alpha$ stable processes can characterize the high peak nature in financial assets, their tail distributions are too thick to capture real tail behaviors in markets. After multiplying tempered functions, they get intermediate between normal distributions and stable distributions appropriately so as to describe the fat tail behaviors of financial data. Then we develop the stochastic volatility Lévy processes (SVLV hereafter) models

* Corresponding author. Tel.: +86 2483679139.

E-mail address: qdgongxiaoli@126.com

(C) 2016. This manuscript version is made available under the Elsevier user license

http://www.elsevier.com/open-access/userlicense/1.0/ 
by subordinating Lévy processes to the stochastic volatility models.

Because model calibrations to market options data formulates a nonlinear optimization problem which often suffers from local minima difficulties, which leads to poor performance in analyzing the behavior of derivative markets, as addressed by $\mathrm{Yu}$ [25]. Therefore, it is significant to obtain an accurate parameter set that calibrates the cross-sectional data well for the proposed model so that the model can be effectively applied to pricing options. The same attitude can be found in Yang and Lee [26]. To verify the performance of global intelligent search method on financial models, we apply Differential Evolution (DE hereafter) algorithm to the newly developed complex models, of which it outperforms local search techniques and other intelligent algorithms illustrated in Fastrich et al. [27] and Zhang et al. [28]. Since the consequences of intelligent search methods can explain the observed leptokurtic features for returns, it performs well in empirical researches. Then simulations are carried out exploiting both model prices and Hangseng index option prices in Chinese Hong kong market case.

The contributions of our work to the previous literatures are that we incorporate both stochastic volatility and infinite Lévy jumps in stock returns simultaneously by subordinating infinite activity Lévy processes to the stochastic volatility models, which are compared to finite jumps stochastic volatility Bates model. The advantage of this newly constructed model framework over the prevailing is the reflection of infinite activity jump behaviors in underlying assets and clustering effect in time changing volatility at the same time. Moreover, the globally intelligent optimization DE algorithm is firstly exploited to calibrate highly dimensional financial models such that the estimation results of SVLV models fitted to option data can be significantly improved, solving the poor performance of nonlinear optimization problems when utilizing local search techniques.

The remainder of the paper is organized as follows. In Section 2 we describe the proposed stochastic volatility Lévy jumps models by subordinating the extensively used infinite activity Lévy processes to the mean reverting CIR stochastic volatility process. In Section 3 we give an outline of Carr-Madan's Fourier transform method and the fast Fourier transform (FFT) technique, the Differential Evolution algorithm is briefly introduced subsequently. Then the empirical researches results are exhibited in Section 4 in which calibrations are performed both to real market data, finally we conclude the paper in Section 5.

\section{Stochastic volatility models with infinite acticity Lévy processes}

\subsection{Infinite acticity Lévy processes}

One can define the Lévy process $X(t)$ in the filtration space $(\Omega, F, P)$ an infinite divisible distribution that has independent and stationary increments and $n$th power of characteristic functions as characteristic functions $\varphi(u)$ themselves. In Sato [29] the infinite divisible Lévy distribution has a triplet of characteristics $(\mu, \sigma, v)$, in which it respectively represents one of the three independent parts: a linear deterministic part, a Brownian motion part and a pure jump part.

In view of the complicated distribution functions expressions for Lévy processes, we generally use the characteristic function $\varphi(u)=E\left[e^{i u X(t)}\right]$, or characteristic exponent $\psi(u)=\log \varphi(u)$ for calculation convenience. The function $\psi(u)$ is called the characteristic exponent that meets the following Lévy-Khintchine formula:

$$
\psi(u)=-i u \mu+\frac{\sigma^{2} u^{2}}{2}-\int_{-\infty}^{+\infty}\left(e^{i u x}-1-i u x_{|x| \leq 1}\right) v d x,
$$

where $\mu \in \mathrm{R}, v$ represents the Lévy measure with $v(\mathrm{R})=\lambda \leq \infty$, which means it happens infinite times jumps in any time interval to measure the arrival rate of jumps.

The Lévy measure is obtained by the fast Fourier transform of the characteristic function that determines the jumps behaviors and jumps structure for density of stochastic distributions, and reflects the jump intensity differences for different processes in different jumps distributions structures. The Lévy measure is associated with its corresponding probability density function, whose width corresponds to the peak of the density distribution, and the height associates with the tail of the distribution. The following are three categories summarized measure formulae for infinite pure Lévy jump processes.

$$
\left\{\begin{array}{l}
V G_{\text {Levy }}=\left\{\begin{array}{l}
\operatorname{Cexp}(G x) /|x| 1_{(x<0)} \\
\operatorname{Cexp}(-M x) / x 1_{(x>0)}
\end{array}\right. \\
N I G_{\text {Levy }}=\frac{\alpha \delta}{\pi|x|} K_{1}(\alpha|x|) e^{\beta x} \\
C T S_{\text {Levy }}=\left\{\begin{array}{l}
C_{-} \exp \left(\lambda_{-} x\right) /|x|^{1+\alpha_{-}} 1_{(x<0)} \\
C_{+} \exp \left(-\lambda_{+} x\right) / x^{1+\alpha_{+}} 1_{(x>0)}
\end{array}\right.
\end{array}\right.
$$

where $K_{1}($.$) is the modified Bessel function of the second kind in NIG Lévy measure.$ 
Model parameters above directly affect the Lévy measure and the probability density distribution in returns. We have chosen CGM representation in VG model, where parameter C determines the aggregate activity rate of the process, while parameters $\mathrm{G}$ and $\mathrm{M}$ respectively controls the arrival rates of jumps in opposite directions. The parameters $\mathrm{G}$ and $\mathrm{M}$ determine the height of the upward and downward jumps, which are exactly what we expect to know about the Lévy measure of VG model. What we can see from the measure of NIG model is that $\beta$ governs the directional premium, it is positively related to the variance, skewness and kurtosis of the density. The shape of function $K_{1}$ governs the jump size premium and the parameter $\delta$ affects the kurtosis of returns distribution. Because of the rich parameters set, it can generate a variety of distribution forms. The scale parameter C in CTS model is related to the kurtosis of the density function, where smaller $\mathrm{C}$ leads to higher peak. While parameters $\lambda_{+}$

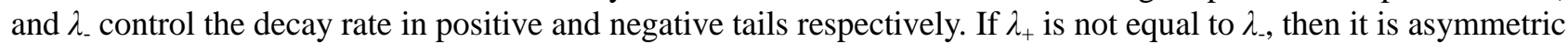
in jumps structure.

\subsection{The stochastic volatility Lévy processes}

The random variation in volatility can alternatively be featured by instantaneous changes in time. The time changes model used to induce stochastic volatility is the integral of the square-root CIR process. The CIR positive mean reverting process serves as a measure of time change rate in which it speeds up or slows down the time elapse rate to increase or decrease the activity intensity so that it can capture the volatility clustering and heteroskedasticity effect. It satisfies the SDE:

$$
d v_{t}=\kappa\left(\eta-v_{t}\right) d t+\lambda \sqrt{v_{t}} d W_{t 2},
$$

where $W=\left\{W_{t 2}, t_{2} \geq 0\right\}$ denotes the standard Brownian motion. $\kappa$ is the speed of mean reversion, $\eta$ is the long term variance, $\lambda$ is the volatility of volatility.

The economic time $V_{t}$ passed in $t$ units of calendar time can be computed by integral of $v_{t}$ between ranges from zero to time $t$, making time stochastic by the stochastic clock built in, whose characteristic function is expressed as follows:

$$
\varphi(u, t)=\frac{\exp \left(\kappa^{2} \eta t / \lambda^{2}\right) \exp (2 v(0) i u /(\kappa+\tau \operatorname{coth}(\tau t / 2)))}{(\cosh (\tau t / 2)+\kappa \sinh (\tau t / 2) / \tau)^{2 \kappa \eta / \lambda^{2}}},
$$

where $\tau=\sqrt{\kappa^{2}-2 \lambda^{2} i u}$

The stochastic volatility Lévy process $S(t)=X(V(t))$ incorporates jumps and stochastic volatility simultaneously, and finally ends up with three resulting models abbreviated as SVVG, SVNIG and SVCTS through subordinating Lévy processes to the stochastic clock. Schoutens [13] demonstrates the characteristic functions for the stock price processes are the following form:

$$
E[\exp (i u S(t))]=E\left[\exp \left(V(t) \psi_{X}(u)\right)\right]=\varphi\left(-i \psi_{X}(u), t, v(0) ; \kappa, \eta, \lambda\right)
$$

The characteristic functions of risk neutral stock price processes are obtained by a mean-correcting factor $\exp ((r-q) t) / E\left[\exp (S(t)) \mid v_{0}\right]$, where $r$ and $q$ refer to constant continuously compound interest rate and dividend yield respectively.

The characteristic function for the SVVG process is as follows:

$$
\varphi_{S V V G}=\exp \left(i u(\log (S(0)+(r-q) t)) \times \frac{\varphi\left(-i \psi_{V G}(u ; 1, G, M), t, C ; \kappa, \eta, \lambda\right)}{\varphi\left(-i \psi_{V G}(-i ; 1, G, M), t, C ; \kappa, \eta, \lambda\right)^{i u}},\right.
$$

where it contains six parameters with $\nu, \theta, \sigma, \kappa, \eta, \lambda$.

The characteristic function for the SVNIG process can be expressed as:

$$
\varphi_{S V C I R}=\exp \left(i u(\log (S(0)+(r-q) t)) \times \frac{\varphi\left(-i \psi_{N I G}(u ; 1, v, \theta), t, \sigma ; \kappa, \eta, \lambda\right)}{\varphi\left(-i \psi_{N I G}(-i ; 1, v, \theta), t, \sigma ; \kappa, \eta, \lambda\right)^{i u}},\right.
$$

in which there are six parameters consisting of $\mathrm{C}, \mathrm{G}, \mathrm{M}, \kappa, \eta, \lambda$ for calibration.

The characteristic function for the SVCTS process is given by:

$$
\varphi_{S V C T S}=\exp \left(i u(\log (S(0)+(r-q) t)) \times \frac{\varphi\left(-i \psi_{C T S}\left(u ; 1, \lambda_{-}, \lambda_{+}, \alpha_{-}, \alpha_{+}, C_{+}\right), t, C_{-} ; \kappa, \eta, \lambda\right)}{\varphi\left(-i \psi_{C T S}\left(-i ; 1, \lambda_{-}, \lambda_{+}, \alpha_{-}, \alpha_{+}, C_{+}\right), t, C_{-} ; \kappa, \eta, \lambda\right)^{i u}},\right.
$$

which is a nine parameters process with $C_{+}, C_{-}, \lambda_{+}, \lambda_{-}, \alpha_{+}, \alpha_{-}, \kappa, \eta, \lambda$.

The stochastic volatility extensions of Lévy processes based models overcome the shortcoming of floating smile property in fitting to option prices. The above models also have such appealing nature that it can find analytical solutions for option prices. Therefore it is convenient for parameters estimation in reasonable amount of time. The analytical solutions can be obtained utilizing characteristic functions and taking FFT technique. 


\section{Option pricing and calibration methodology}

\subsection{FFT for European option pricing}

The SVLV models permit utilizing Fourier transform methods for option pricing because of the availability of analytical characteristic functions of SVLV models, thereby realizing calibration to real market data. Carr and Madan [30] developed the general option pricing method which can be used conveniently when characteristic functions of the risk neutral asset price process are available. And it is applied in pricing foreign equity option with stochastic volatility in Ref. [31]. Let $C(K, T)$ denote the European call option price with strike $K$ and time to maturity $T$, and $\alpha$ be a positive constant assuring the existence of the asset price's $\alpha$ th moment. The formula is given by:

$$
C(K, T)=\frac{\exp (-\alpha \log (K))}{\pi} \int_{0}^{+\infty} \exp (-i u \log (K)) \xi(u) d u,
$$

where $\xi(u)=\frac{\exp (-r T) \phi(u-(\alpha+1) i, T)}{\alpha^{2}+\alpha-u^{2}+i(2 \alpha+1) u}$.

Let $k$ denote the log of the strike price $K$, the fast Fourier Transform technique can be utilized to expedite the calculation speed. The FFT returns $N$ values of $k$ and for a regular spacing size of $z$ where $N$ is a power of 2, the value for $k$ is $k_{u}=-b+z(a-1)$, with $\alpha$ being an positive integer and $b=N z / 2$. We use the FFT method to (9) that is an $O(N \ln N)$ algorithm for computing $w(u) \approx \sum_{j=1}^{N} e^{-i \frac{2 \pi}{N}(j-1)(u-1)} x(j), u=1, \ldots, N$. The strength of FFT is that it goes efficiently in pricing several options with the same maturity.

Employing the Trapezoid rule for the integral in the above formula (9), it yields:

$$
C\left(k_{u}\right) \approx \frac{\exp \left(-\alpha k_{u}\right)}{\pi} \sum_{j=0}^{N} \exp (-i z v(j-1)(a-1)+i b v(j-1)) \xi(v(j-1)) v .
$$

In the FFT application, we note that $v z=2 \pi / N$, let $u_{j}=v(j-1)$, with Simpson's rule weightings, the value of European call option is derived:

$$
C\left(k_{u}\right) \approx \frac{\exp \left(-\alpha k_{u}\right)}{\pi} \sum_{j=1}^{N} \exp \left(-i \frac{2 \pi}{N}(j-1)(a-1)+i b u_{j}\right) \xi\left(u_{j}\right) \frac{v}{3}\left[3+(-1)^{j}-\ell_{j-1}\right],
$$

where $\ell_{n}$ is the Kronecker delta function that is unity for $n=0$ and zero otherwise.

\subsection{Differential Evolution algorithm}

Because when calibrating the above computational demanding SVLV models to real market option data, it develops a nonlinear problem which often goes slowly, inefficiently, and easily falls into local optimum exploiting traditional optimization techniques [32-34]. Therefore, it is significant to bring efficient globally optimized technique to obtain good parameters sets. The recently emerging evolution algorithms are particularly suitable to solve optimization problems encountered in the calibration of financial models. It has been proven that poor local solutions of calibration generate undesirable fitting results and the necessity to introduce global intelligent search methods to enhance the quality of calibration procedures. The Differential Evolution (DE) algorithm avoids becoming trapped in flat regions or local optima of the objective function. A comprehensive description of DE is provided by Price et al. [35]. Application of DE in the portfolio optimization context is in Krink and Paterlini [36]. The basic idea is to evolve a population of solutions by means of recombination, crossover, and survival of the fittest, and the basic steps are as follows.

1. Population initialization. The algorithm starts with the gth generation of a current population $N$ of $d$ dimensional random vector. Each individual $I_{i}^{\mathrm{g}}$ is a set of elements that characterizes a portfolio, which includes the auxiliary vector and its corresponding portfolio weight vector.

$$
I_{i, k}^{g} \leftarrow I_{\text {min }, k}+\operatorname{Random}(0,1)\left(I_{\max , k}-I_{\text {min, }, k}\right),
$$

where $I_{\max , k}$ and $I_{\min , k}$ denote the $k$ th dimensional maximum and minimum values of $I_{i}^{\mathrm{g}}$. $\operatorname{Random}(0,1)$ refers to the random numbers generated in $(0,1)$.

2. Mutation step. For an individual with $k$ dimension, three different individuals are randomly chosen from the current population. In each generation, the algorithm creates a candidate solution for each existing solution. This new solution or mutation vector $v_{i}^{\mathrm{g}}$ is created by adding the difference, weighted by a scaling factor $F$ between two other solutions $I_{i,}{ }_{k}{ }_{k}$ to a third solution, which is shown by:

$$
v_{i, k}^{g} \leftarrow I_{i 1, k}+F\left(I_{i 2, k}^{g}-I_{i 3, k}^{g}\right) .
$$


3. Crossover step. Then an element-wise crossover takes place with probability $P c$ and a uniform random vector $r$ between this 'auxiliary' solution $v_{i}^{\mathrm{g}}$ and the existing solution $I_{i}^{\mathrm{g}}$ to produce the diversified intermediate individual vector $m_{i}^{\mathrm{g}}$, which follows:

$$
m_{i, k}^{g} \leftarrow\left\{\begin{array}{ll}
v_{i, k}^{g}, & \text { if } r \leq P c \\
I_{i, k} & \text { else }
\end{array} .\right.
$$

4. Selection step. Calculate the value of objective function $f$ that serves as measures of fitness level. If this final candidate solution $m_{i}^{\mathrm{g}}$ is better than $I_{i}^{\mathrm{g}}$, it replaces it; if not, the old solution is kept. Or to say, whenever the objective function value of the new individual is smaller than that of the current individual, the latter is replaced by the former, which subjects to:

$$
I_{i}^{g+1} \leftarrow \begin{cases}m_{i}^{g}, & \text { if } f\left(m_{i}^{g}\right) \leq f\left(I_{i, k}\right) \\ I_{i, k} & \text { else }\end{cases}
$$

5. Convergence. This evolution of the population keeps the individuals moving through the search space until they assemble around the best solution that have found. The algorithm is terminated by a pre-specified stopping criterion.

\section{Empirical results}

\subsection{Statistical description of index returns}

The original data are taken from stock markets, which are Hang Seng Index (HSI) and Standard \& Poor 500 Index (SP500) that are two important indexes representing the global capital markets. The selected closing prices of HSI and SP500 indexes are from the Wind database, with sample interval ranging from 2011 to January, 2015, totally 1000 samples. Table 1 is the descriptive statistics of the sample returns. And Fig. 1 displays the volatility paths, skewness movements, kurtosis changes sequences and noise sequences of HSI (upper panel) and SP500 (lower panel) from left to right respectively.

Table 1

Descriptive statistics of index returns.

\begin{tabular}{ccccccccc}
\hline index & mean & variance & skewness & kurtosis & Correlation (10) & heteroskedasticity & $J-B$ test & $(\mathrm{p}$ value) \\
\hline HSI & -0.0006 & 0.0012 & -0.0824 & 6.28 & 19.8 & 235.3 & $1.86 \mathrm{e}-4$ \\
SP500 & $6.91 \mathrm{e}-3$ & 0.0103 & -0.1255 & 7.4237 & 24.6 & 240.6 & $2.02 \mathrm{e}-4$ \\
\hline
\end{tabular}
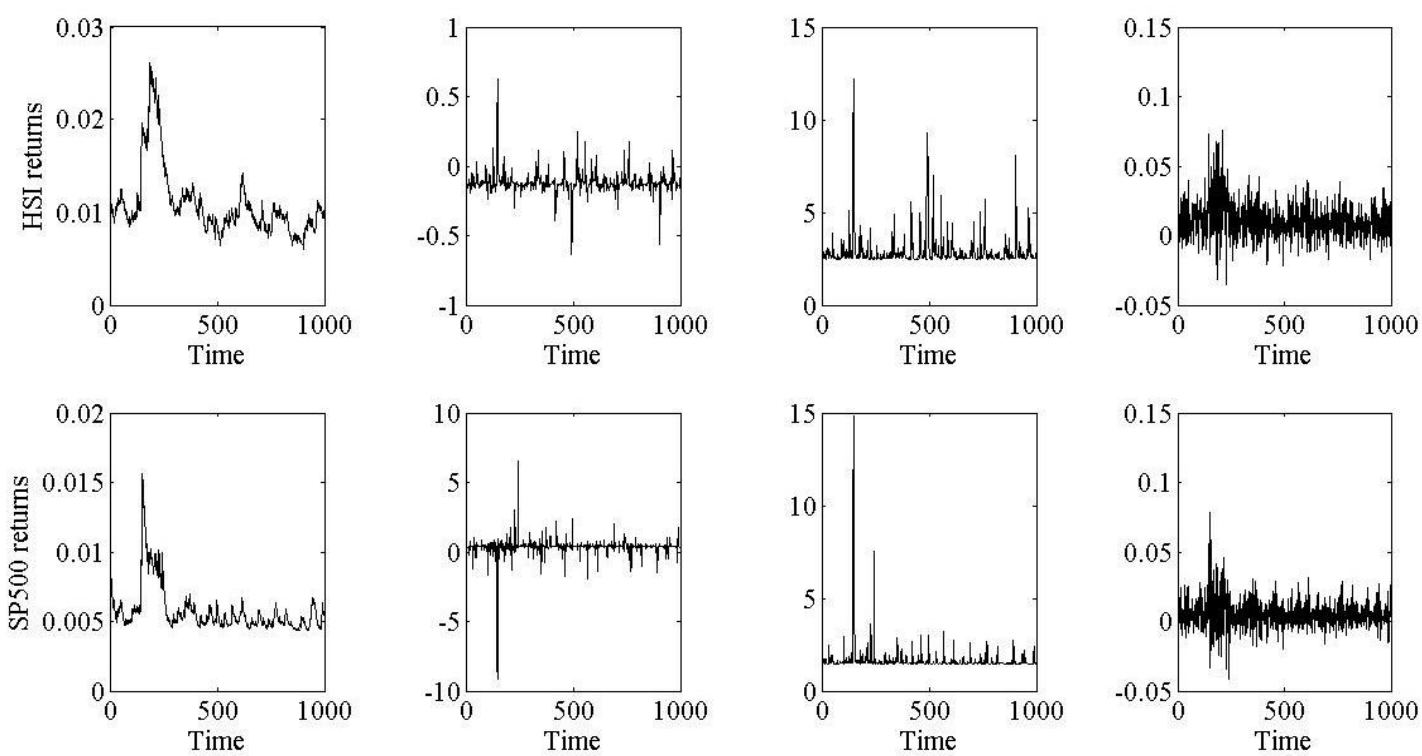

Fig. 1. The sequence of volatility, skewness, kurtosis and noises from left to right

The statistics in Table 1 reveal that the HSI and SP500 indexes return distributions exhibit asymmetry and high peak in 2011 to January 2015, with 10 orders autocorrelation coefficient significant. Heteroscedasticity test results show remarkable heteroscedasticity effect in both HSI and SP500 indexes returns, the $J-B$ normality test 
rejects the null hypothesis of normal distribution, indicating that the index returns are not normally distributed. The values of skewness and kurtosis once again verify the leptokurtosis nature and asymmetry feature existed in HSI index and SP500 index.

Fig. 1 shows outstanding volatility agglomeration in the time series, as well as volatility persistence and mean reversion tendency in volatility processes. The timely evolution process of volatility can reflect irregular infinite jumps in stock prices dynamics. There are obviously many singular values in the noise sequences, the prominent negatively biased curves illustrate the time series data exist left skew. The skewness processes directly validate the left skew phenomenon of both indexes series. The kurtosis value changes are always greater than that in normal distribution, which shows leptokurtosis exists in two indexes. Table 1 and Fig. 1 collectively illustrate the non-Gaussian features of financial returns.

From comparisons in Fig. 1 we find that HSI returns are more volatile than SP500 returns, with changes of volatility and skewness sequences happening more frequently and unpredictably. It is noteworthy that there exist extreme positive jumps stages during the end of 2011, what makes differences are that the HSI skewness sequence emerges shortly persistent positive values while SP500 skewness emerges deep negative values. This is probably due to the overestimation of risks in HongKong financial markets and underestimation of risks in American markets. The appearance frequency of extra kurtosis of HSI is larger than that of SP500, indicating that the probability of extreme events in emerging financial markets is much more likely than that in mature financial markets, whereas the average kurtosis value 7.4237 of SP500 is larger than that of HSI 6.28, meaning more severe volatility degree in mature markets once crisis happens.

\subsection{Calibrating model parameters by $D E$}

We consider options on the HSI traded on Thursday 9th April 2015, on which day options with maturity longer than one hundreds days are ruled out for their low trade volume. The HSI on that day is 26944. Calibrating an option pricing model means searching parameters sets meeting conditions that the model's prices are consistent with their market prices, which will result in an optimization problem of the objective function. Here we choose the root mean square error (RMSE) to measure the performance of option pricing. Alternatively, it can employ the average absolute percentage error (APE) as a measure.

$$
\begin{aligned}
& R M S E=\sqrt{\frac{1}{N} \sum_{i=1}^{N}\left[C_{\text {mark }}(i)-C_{\text {model }}(i)\right]^{2}}, \\
& A P E=\frac{1}{C_{\text {aver }}} \sum_{i=1}^{N} \frac{\left|C_{\text {mark }}(i)-C_{\text {model }}(i)\right|}{N},
\end{aligned}
$$

where $N$ is the number of market options, $C_{\text {mark }}, C_{\text {model }}$ and $\mathrm{C}_{\text {aver }}$ denote option prices for the markets and models, and average option prices respectively.

The objective function is given by RMSE between the market observables and the model prices, and is taken over all strikes and maturities. We list the calibration results for different models obtained by applying the Differential Evolution algorithm in Table 2, and add the results of Bates model for comparative purpose. Fig. 2 displays the convergence curves of intermediate objective function applied to SVCTS model by DE and traditional SOP local method for simplicity, with other models figures similar.

Table 2

\begin{tabular}{|c|c|c|c|c|c|c|c|c|c|}
\hline \multirow{2}{*}{$\begin{array}{c}\text { Model } \\
\text { Bates }\end{array}$} & \multicolumn{9}{|c|}{ Parameters } \\
\hline & $\begin{array}{c}v_{0} \\
0.2174\end{array}$ & $\begin{array}{c}\kappa \\
6.4598\end{array}$ & $\begin{array}{c}\eta \\
0.0601\end{array}$ & $\begin{array}{c}\lambda \\
0.2433\end{array}$ & $\begin{array}{c}\rho \\
-0.5783\end{array}$ & $\begin{array}{c}\mu_{J} \\
-0.6325\end{array}$ & $\begin{array}{c}\sigma_{J} \\
2.1849\end{array}$ & $\begin{array}{c}\omega \\
157.0251\end{array}$ & \\
\hline SVVG & $\begin{array}{c}\mathrm{C} \\
12.1609\end{array}$ & $\begin{array}{c}\mathrm{G} \\
15.0435\end{array}$ & $\begin{array}{c}\mathrm{M} \\
21.1629\end{array}$ & $\begin{array}{c}\kappa \\
7.0526\end{array}$ & $\begin{array}{c}\eta \\
0.0477\end{array}$ & $\begin{array}{c}\lambda \\
0.5017\end{array}$ & & & \\
\hline SVNIG & $\begin{array}{c}v \\
20.2086\end{array}$ & $\begin{array}{c}\theta \\
-6.4831\end{array}$ & $\begin{array}{c}\sigma \\
2.1956\end{array}$ & $\begin{array}{c}\kappa \\
6.9541\end{array}$ & $\begin{array}{c}\eta \\
00538\end{array}$ & $\begin{array}{c}\lambda \\
0.4721\end{array}$ & & & \\
\hline SVCTS & $\begin{array}{c}C_{+} \\
2.6142\end{array}$ & $\begin{array}{c}C . \\
0.8793\end{array}$ & $\begin{array}{c}\lambda_{+} \\
27.5201\end{array}$ & $\begin{array}{c}\lambda_{-} \\
19.5416\end{array}$ & $\begin{array}{c}\alpha_{+} \\
0.0213\end{array}$ & $\begin{array}{c}\alpha \\
0.4875\end{array}$ & $\begin{array}{c}\kappa \\
8.1284\end{array}$ & $\begin{array}{c}\eta \\
0.032\end{array}$ & $\begin{array}{c}\lambda \\
0.3684\end{array}$ \\
\hline
\end{tabular}

Model calibration results

What we can see from the Bates model in Table 2 is that the jump component has significant intensity and a relatively smaller expectation value. This implies that the compound Poisson process attempts to jump as an infinite activity process. The correlation coefficient $\rho<0$ confirms the leverage effect existed in volatility jumps and stock prices. The valuation of volatility reverting rate $\kappa$, variance of volatility $\eta$ in infinite models are smaller than those in Bates, while the long term mean of volatility $\lambda$ is higher than that in Bates. This is because the infinite activity models can capture infinite small jumps and extremely large jumps simultaneously, thus reducing the persistence of the volatility diffusion process. The value of shape parameter $\alpha$ indicates leptokurtic nature and 
fat tail phenomenon in returns. The density decay rate of right tail $\lambda_{+}=27.5201$ is larger than the left one $\lambda_{-}=19.5416$, implying left skew feature in density distribution of the stochastic process. The negative skew is also captured by other two SVLV models, which are reflected by negative estimation of $\theta$ for SVNIG model, lower value of $\mathrm{G}$ than $\mathrm{M}$ for SVVG model.

In the calibration procedure, we define the crossover probability $P c=0.8$, the scaling factor $F=0.85$ and the maximum number of iterations is 200. Fig. 2 shows the convergence curves of the Differential Evolution algorithm applied to SVCTS compared with sequential quadratic programming (SQP) local search method, in which we can see that it takes roughly 120 generations to reach a stable minimum value for DE, whereas it requires approximately 170 generations for SQP to converge. It can be easily found that the DE algorithm converge faster than SQP in speed, as well as getting lower RMSE error value 1.722 than that 2.4965 in SQP. Practically, the running of SQP local search method performs badly in calibration since it has so wide ranges of parameters. It is the DE algorithm that clearly produces potentially reasonable results.

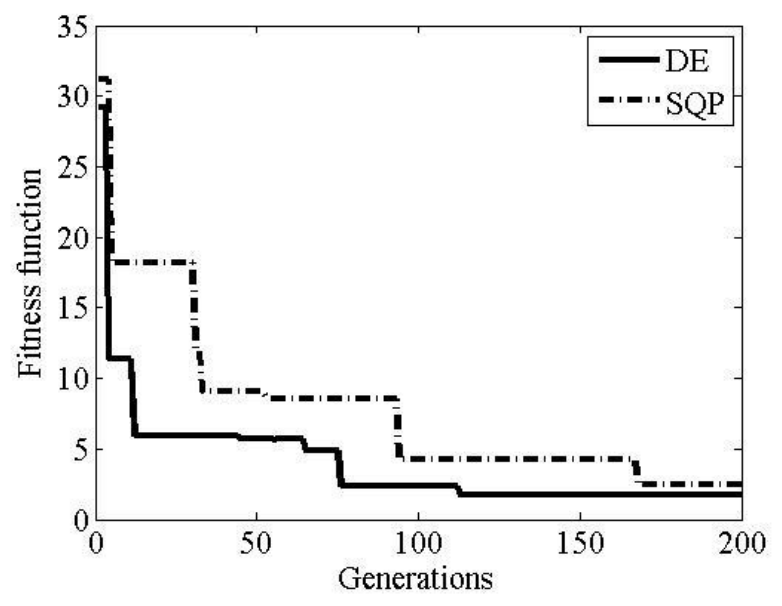

Fig. 2. Convergence curves of DE and SQP applied to SVCTS

To further demonstrate the effectiveness of density distributions fitting, we use the obtained parameters above and the same time series data in section 4.1 to separately draw the density distributions of BS model, Bates model, SVVG model, SVNIG model and SVCTS model compared with the historical density curve. It can be shown that in Fig. 3 the SVCTS model is superior to other infinite activity jump models and finite jump model at the leptokurtosis, which is closer to real phenomenon of returns in financial assets, and more accurately reflect the distribution property of capital markets.

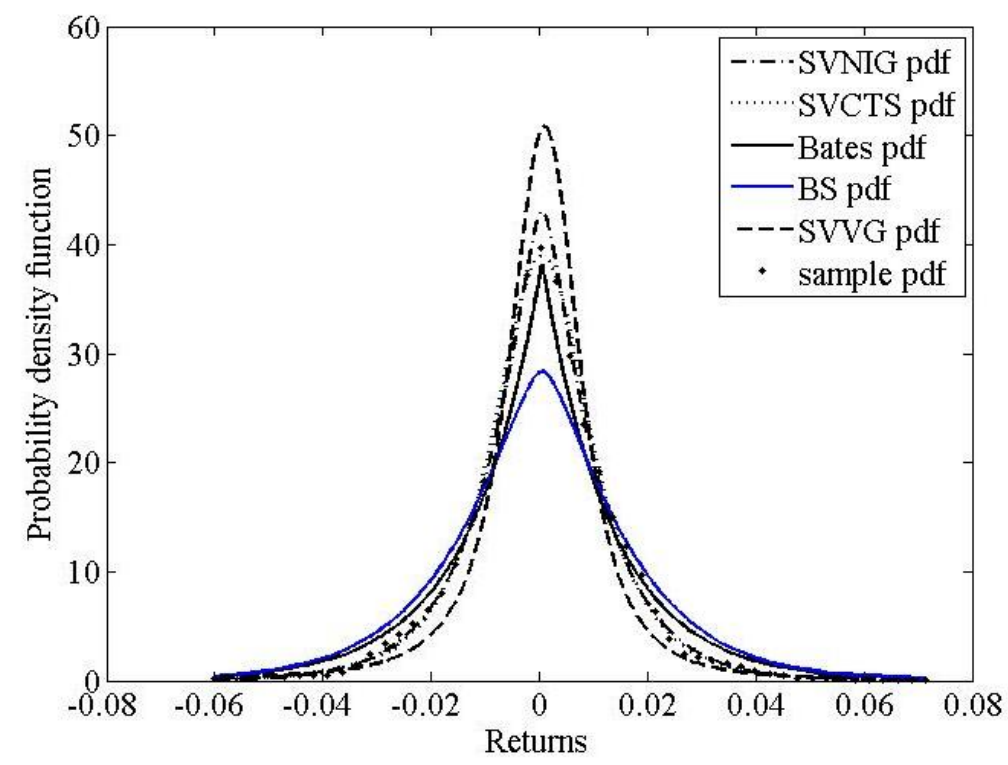

Fig. 3. Density distributions for option pricing in different models

\subsection{Option pricing performance}

It is strongly recommended to conduct out-of-sample forecast to check the robustness of the empirical consequences since unreasonable models may also achieve good results by over-fitting. We perform forecast on 
the next day to the day in calibration, namely Friday 10th April 2015, similarly only short term maturities and medium term maturities options are considered by maturity classifications and moneyness classifications. The HSI on that day is 27272. For comparative purpose, we exhibit the values of RMSE and APE for wide ranges of models as measures of pricing errors.

Using the risk-neutral parameters obtained in the previous day, we calculate the out-of-sample pricing errors such as APE and RMSE for various models specifications. Table 3 shows the superiority of newly constructed SVLV models over BS and Bates models overall with lowest errors. Specifically, for the ATM and OTM options, SVLV models considerably reduce pricing errors for short maturities and medium maturities compared to BS and Bates models. It implies that stochastic volatility model combining infinite Lévy jumps adds explanatory power for improving empirical fitting of HSI call options. However, for the ITM options, these results are relatively consistent in general since the positive intrinsic values of options. For example, the APE of BS model is $1.42 \%$, 9.73\%, 1.89\% less than SVVG, SVNIG and SVCTS models respectively for short maturity ITM options, while for OTM options, the APE of BS model is $42.3 \%, 44.43 \%, 48.49 \%$ more than SVVG, SVNIG and SVCTS models respectively, showing the usefulness of infinite jumps Lévy models in OTM option prediction. The same effect is shown in RMSE calculation. The RMSE of SVLV models are $20.32 \%, 16.47 \%, 10.48 \%$ less than finite activity Bates models for medium maturity ATM options, with $13.43 \%, 14.47 \%, 22.22 \%$ less than corresponding short maturity option values, therefore SVLV models are suitable for long term maturity options pricing. We see that the SVCTS is a consistently good performer across both moneyness and maturities, with option prices generated by SVCTS model have smaller errors than other models, which indicates that the SVCTS model performs best above all.

Table 3

Pricing errors in moneyness and maturity categories

\begin{tabular}{|c|c|c|c|c|c|}
\hline \multirow{3}{*}{ Models } & \multirow{3}{*}{ Moneyness } & \multicolumn{4}{|c|}{ Call options } \\
\hline & & \multicolumn{2}{|c|}{ Short maturity } & \multicolumn{2}{|c|}{ Medium maturity } \\
\hline & & APE & RMSE & APE & RMSE \\
\hline \multirow{4}{*}{ BS } & ITM $(<0.97)$ & 3.2754 & 5.0824 & 4.0159 & 5.1436 \\
\hline & $\operatorname{ATM}(0.97-1.03)$ & 6.2533 & 8.6792 & 8.4216 & 6.7411 \\
\hline & $\mathrm{OTM}(>1.03)$ & 7.7692 & 6.3556 & 9.6973 & 7.8001 \\
\hline & ITM $(<0.97)$ & 3.6274 & 5.1231 & 4.7135 & 4.9519 \\
\hline \multirow[t]{3}{*}{ Bates } & $\operatorname{ATM}(0.97-1.03)$ & 5.8072 & 7.4946 & 8.1635 & 6.0122 \\
\hline & $\mathrm{OTM}(>1.03)$ & 7.0226 & 6.5163 & 7.3493 & 6.3080 \\
\hline & ITM $(<0.97)$ & 3.6655 & 6.0255 & 3.1406 & 5.8822 \\
\hline \multirow[t]{3}{*}{ SVVG } & $\operatorname{ATM}(0.97-1.03)$ & 5.6209 & 5.4337 & 5.3726 & 4.7905 \\
\hline & $\mathrm{OTM}(>1.03)$ & 4.4828 & 4.8632 & 6.1233 & 4.1372 \\
\hline & ITM $(<0.97)$ & 3.3002 & 5.7539 & 4.6928 & 5.1034 \\
\hline \multirow[t]{3}{*}{ SVNIG } & $\operatorname{ATM}(0.97-1.03)$ & 4.3172 & 5.7484 & 6.0529 & 5.0219 \\
\hline & $\mathrm{OTM}(>1.03)$ & 3.0571 & 4.9536 & 5.7189 & 4.8862 \\
\hline & ITM $(<0.97)$ & 3.7961 & 4.9371 & 2.9508 & 4.7603 \\
\hline \multirow[t]{2}{*}{ SVCTS } & $\operatorname{ATM}(0.97-1.03)$ & 3.6281 & 5.5016 & 5.9951 & 5.3820 \\
\hline & $\mathrm{OTM}(>1.03)$ & 4.0022 & 4.7752 & 5.0247 & 4.5623 \\
\hline
\end{tabular}

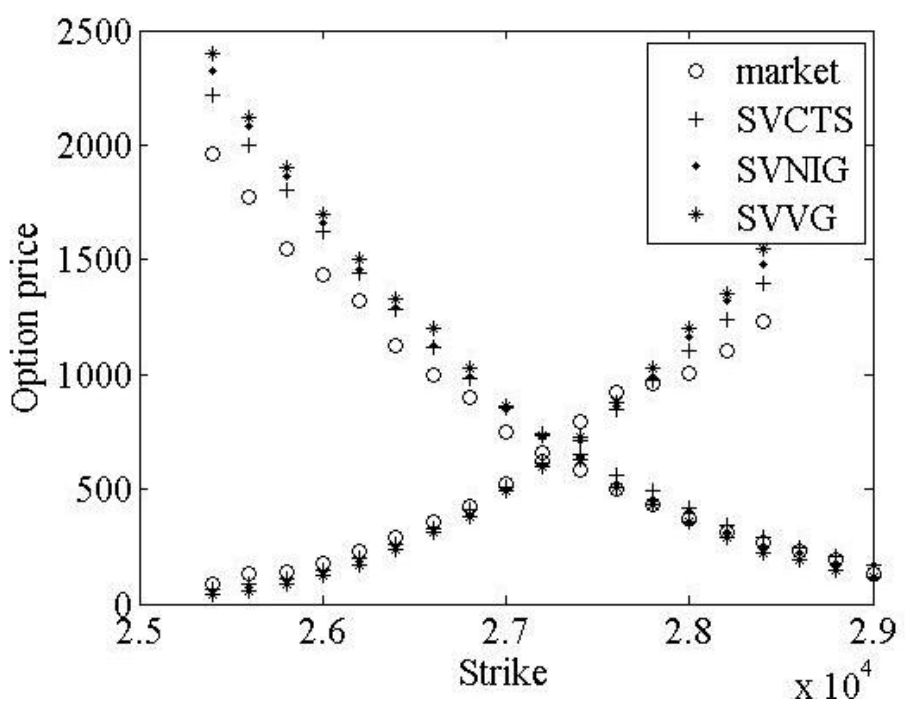

Fig. 4. Out-of-sample option pricing performance 
Fig. 4 is the pricing performance applied to HSI short maturities call options using three kinds of SVLV models, which we have demonstrated their advantages in prediction. It can be observed that the model implied option prices in SVCTS have the smallest deviations between market options and model options, and are perfectly identical with market prices at the money. Consequently, it once again verifies the conclusion drawn from the table. The put options can be readily acquired using the put-call parity.

\section{Conclusion}

The failure of traditional B-S option pricing model to incorporate volatility smile and excess kurtosis has led to various alternative models. We combine stochastic volatility and infinite activity Lévy processes in newly developed SVLV models framework with normal innovations replaced by Lévy innovations, where both complementary strengths of these processes can be utilized to capture asymmetry, leptokurtosis and thicker tail properties in returns in addition to characterizing persistence effect and heteroskedasticity effect in volatility. Moreover, the intelligent optimization DE algorithm is applied to search global optimal solution in calibrating the model parameters through characteristic functions based FFT calculation and is compared to that of local search method to validate the superiority of the DE method.

It is useful to compare these wide ranges of models including Bates, SVVG, SVNIG and SVCTS models on common data sets encompassing both real returns data and options data. Empirical researches have demonstrated that the DE optimization method systematically has better performance in convergence speed and accuracy. The empirical study also indicates that infinite activity SVLV models are preferable to jump-diffusion model in capturing the leptokurtic feature and jumps behaviors despite the presence of diffusion components. Comparing the presentations of three stochastic volatility Lévy processes reveals that the SVCTS model has the smallest pricing deviations as well as a better fitting accuracy to observables. Investigating the hedging performance of the SVLV models employing the DE intelligence method is left for further investigation.

\section{Acknowledgments}

We sincerely thank our anonymous referee. We also would like to acknowledge the financial support from the National Science Foundation of China (No. 71171042).

\section{References}

[1] X. T. Wang, Z. F. Zhao, X. F. Fang, Option pricing and portfolio hedging under the mixed hedging strategy, Physica A 424 (2015) 194-206.

[2] H. R. Ranjbar, A. Seifi, A path-independent method for barrier option pricing in hidden Markov models, Physica A 440 (2015) $1-8$.

[3] A. S. Yacine, J. Jean, Estimating the degree of activity of jumps in high frequency data, Ann. Statist. 37 (2009) $2202-2244$.

[4] S. Klingler, Y. S. Kim, T. Svetlozar, Option pricing with time-changed Lévy processes, Appl. Financ. Econ. 23 (2013) 1231-1238.

[5] S. G. Kou, H. Wang, Option pricing under a double exponential jump diffusion model, Manag. Sci. 50 (2004) $1178-1192$.

[6] S. Mozumder, G. Sorwar, K. Dowd, Option pricing under non-normality: a comparative analysis, Rev. Quan. Financ. Acc. 40 (2013) 273-292.

[7] Q. H. Shi, X. P. Yang, Pricing Asian options in a stochastic volatility model with jumps, Appl. Math. Comput. 228 (2014) 411-422.

[8] I. Abdelrazeq, Model verification for Lévy-driven Ornstein-Uhlenbeck processes with estimated parameters, Stat. Probab. Lett.104 (2015) 26-35.

[9] P. Carr, L. Wu, Time-changed Lévy processes and option pricing, J. Financ. Econ. 71 (2004) 113-141.

[10] B. Podobnik, P.Ch. Ivanow, I. Grosse, K.Matia, H.E. Stanley, ARCH-GARCH approaches to modeling high-frequency financial data, Physica A 344 (2004) 216-220.

[11] W.L. Xiao, W.G. Zhang, X.L. Zhang, X.Y. Chen, The valuation of equity warrants under the fractional Vasicek process of the short-term interest rate, Physica A 394 (2014) 320-337.

[12] V. Todorov, Econometric analysis of jump-driven stochastic volatility models, J. Econometrics, 160 (2011) 12-21.

[13] K. A. Andreas, Carol, Stochastic volatility jump-diffusions for European equity index dynamics, Eur. Financ. Manag. 19 (2013) $470-496$.

[14] J. X. Huang, W. L. Zhu, X. F. Ruan, Option pricing using the fast Fourier transform under the double exponential jump model with stochastic volatility and stochastic intensity, J. Comput. Appl. Math. 263 (2014) 152-159.

[15] D. S. Bates, Jumps and stochastic volatility: Exchange rate processes implicit in deutsche mark options, Rev. Financ. Stud. 9 (1996) 69-107.

[16] S. L. Heston, A closed form solution for options with stochastic volatility with applications to bond and currency options, Rev. Financ. Stud. 6 (1993) 327-343.

[17] W. Schoutens, S. Symens, The pricing of exotic options by Monte-Carlo simulations in a Levy market with stochastic volatility, Int. J. Theor. Appl. Finance 6 (2003) 839-864.

[18] D. Madan, P. Carr, E. Chang, The variance gamma process and option pricing, Eur. Financ. Rev. 2 (1998) $79-105$.

[19] O. E. Barndorff-Nielsen, Processes of normal inverse Gaussian type, Financ. Stoch. 2 (1997) 41-68.

[20] J. Rosinski, Tempering stable processes, Stoch. Proc. Appl. 117 (2007) 677-707. 
[21] P. Carr, H. Geman, D. B. Madan, M. Yor, The fine structure of asset returns: An empirical investigation, J. Business, 75 (2002) 205-332.

[22] Y. S. Kim, S. Rachev, M. Bianchi, Time series analysis for financial market meltdowns, J. Bank. Finance 35 (2011) $1879-1891$.

[23] U. Kuchler, S. Tappe, Tempered stable distributions and processes, Stoch. Process Appl. 123 (2013) 4256-4293.

[24] T. S. Zaevski, Y. S. Kim, F. J. Fabozzi, Option pricing under stochastic volatility and tempered stable Lévy jumps, Int. Rev. Financ. Anal. 31 (2014) 101-108.

[25] C. L. Yu, H. T. Li, M. T. Wells, MCMC estimation of Levy jump models using stock and option prices, Math. Finance 21 (2011) 383-422.

[26] S. Yang, J. Lee, Multi-basin particle swarm intelligence method for optimal calibration of parametric Levy models, Expert Sys. Appl. 39 (2012) 482-493.

[27] B. Fastrich, P. Winker, Combining forecasts with missing data: making use of portfolio theory, Comput. Econ. 44 (2014) $127-152$.

[28] W. G. Zhang, Y. J. Liu, W. J. Xu, A new fuzzy programming approach for multi-period portfolio optimization with return demand and risk control, Fuzzy Sets and Sys. 246 (2014) 107-126.

[29] K. Sato, Lévy Processes and Infinitely Divisible Distributions, Cambridge University Press, Cambridge, 1999.

[30] P. Carr, D. Madan, Option valuation using the fast Fourier transform, J. Comput. Finance 2 (1998) 61-73.

[31] Q. Sun, W. D. Xu, Pricing foreign equity option with stochastic volatility, Physica A 437 (2015) 89-100.

[32] C. L. Su, S. M. Yang, W. L. Huang, A two-stage algorithm integrating genetic algorithm and modified Newton method for neural network training in engineering systems. Expert Sys. Appl. 38 (2011) 12189-12194.

[33] M. N. Mathew, A new gradient based particle swarm optimization algorithm for accurate computation of global minimum. Appl Soft Comput. 12(2012) 353-359.

[34] Z. B. Li, K. F. C. Yiub, Z. G. Feng, A hybrid descent method with genetic algorithm for microphone array placement design. Appl. Soft Comput. 13 (2013) 1486-1490.

[35] K. Price, R. Storn, J. Lampinen, Differential evolution. A practical guide to global optimization, Natural computing series, Berlin: Springer, 2005.

[36] T. Krink, S. Paterlini, Multiobjective optimization using differential evolution for real-world portfolio optimization, Comput. Manag. Sci. 8 (2011) 157-179. 